\title{
Tracking energy fluctuations from fragment partitions in the Lattice Gas model
}

\author{
F. Gulminell:* \\ LPC Caen (IN2P3 - CNRS / EnsiCaen et Université), F-14050 Caen Cédex, France \\ Ph.Chomaz \\ GANIL ( DSM - CEA / IN2P3 - CNRS), B.P.5027, F-14076 Caen Cédex 5, France \\ M.D'Agostino \\ Dipartimento di Fisica and INFN, Bologna, Italy
}

\begin{abstract}
Partial energy fluctuations are known tools to reconstruct microcanonical heat capacities. For experimental applications, approximations have been developed to infer fluctuations at freeze out from the observed fragment partitions. The accuracy of this procedure as well as the underlying independent fragment approximation is under debate already at the level of equilibrated systems. Using a well controlled computer experiment, the Lattice Gas model, we critically discuss the thermodynamic conditions under which fragment partitions can be used to reconstruct the thermodynamics of an equilibrated system.
\end{abstract}

\section{INTRODUCTION}

The observation of abnormal fluctuations in nuclear multifragmentation and its possible connection to a negative heat capacity [1] has raised much interest and discussions in the last years [2, 3, 4, 5, 6, 7].

For a microcanonical ensemble, it has been proposed in ref. 8] that the kinetic energy fluctuation $\sigma_{K}^{2} / T^{2}$ can be used to reconstruct the heat capacity even in the context of phase transitions and for small systems. The accuracy of the fluctuation expression has been successfully tested on numerical experiments on the liquid gas phase transition, using the microcanonical Lattice Gas model [9] and molecular dynamics simulations with a Lennard-Jones potential [5, 10].

From the experimental point of view, it has been proposed to use the clusters asymptotically detected in nuclear multifragmentation reactions to backtrace the fluctuations of the total energy partitioning at freeze-out 1]. The robustness of the experimental procedure has been tested in ref. [11]. In particular, statistical models have been used to generate events, then analyzed using the experimental procedure and a good reproduction of the model heat capacities has been reported [11]. Using molecular dynamics at equilibrium and recognizing fragments through the Hill algorithm 12 the authors of ref. 5] have come to opposite conclusions criticizing the independent fragment hypothesis on which is based the experimental method as well as the zero temperature approximation for the fragment binding used to evaluate the fragment internal interaction energy. The model of ref. [5] has also been studied in ref. [10], where the dynamics of the expansion was explicitly included. The result was that the fluctuations of dense systems were strongly modified by the dynamics and that only the latest phase

${ }^{*}$ member of the Institut Universitaire de France of the expansion, after a freeze-out corresponding to dilute configurations, can be accessed from the observation of asymptotic partitions.

More generally, the question of the thermodynamic conditions under which the energy partitioning of a small fragmenting system can be retraced from the measured fragment sizes and kinetic energies, raises important questions for the whole field of nuclear thermodynamics. Indeed the independent fragment hypothesis is not only needed to reconstruct partial energy fluctuations [1] but is also necessary for any other quantitative estimation of the nuclear phase diagram [14]. Moreover only if the fragmenting source can be approximated by an ensemble of non- (or weakly-) interacting nuclear clusters, the statistical models 15 that have successfully reproduced heavy ion data since two decades can be theoretically justified.

To contribute to this debate, we want to address the problem of the independent fragment hypothesis and of the fragment energetics at equilibrium in the framework of a well controlled exact numerical model, the Lattice Gas model[9]. We will show in this article that, in the Lattice Gas model, the system heat capacity can be well estimated from fragment sizes at all energies and for all pressures $p / p_{c} \lesssim 1 / 3$ almost independent of the parametrization adopted for the fragment energies. For higher pressures this approximation tends to break down, but the estimation of fluctuation stays at the $30 \%$ accuracy level even in the supercritical regime.

\section{LATTICE GAS RESULTS}

It has been proposed in [8] to use the partitioning of a fixed total energy $\left(E_{t o t}\right)$ into kinetic $(K)$ and configurational $(V)$ energies, $E_{t o t}=K+V$, in order to look for entropy curvature anomalies. Indeed, for classical systems with momentum independent interactions, because of the microstates equiprobability the microcanonical dis- 
tribution of $K$ at a fixed energy $E_{t o t}$ reads

$$
P_{E}(K)=\exp \left(S_{K}(K)+S_{V}(V)-S_{t o t}\left(E_{t o t}\right)\right)
$$

where $S_{K}, S_{V}, S_{t o t}$ are the kinetic, potential, and total entropies. If this distribution is normal, a gaussian approximation can be performed leading to analytic expressions relating temperatures and heat capacities to the observed moments of the distribution. When only the leading order is kept, we recover the simplest expression used in ref. [16 to relate the total microcanonical heat capacity $C$ to the kinetic one $C_{K}$ and the kinetic energy fluctuation $\sigma_{K}$ scaled by the system temperature $T$ [17]

$$
C=C_{K}\left(1-\frac{\sigma_{K}^{2}}{C_{K} T^{2}}\right)^{-1}
$$

Even if in practice it seems that Eq. (1) is often accurate enough, the validity and the accuracy of this approximate expression should always be checked by controlling the actual distribution and when the distribution is normal by evaluating the corrections. In our work this has been done both in theory [8] and experiments [11]. The accuracy of the simple expression (II) to infer the heat capacity even in the vicinity of a phase transition, or worse of a critical point is also verified in refs. [5, 10].

The application to experimental data of the idea proposed in [8] requires the development of different tools. Let us first briefly recall the problem of the potential energy determination for an ensemble of fragments. If we look at a system of $A$ interacting particles as a system of $M_{f}$ clusters (including monomers), the potential energy $V=\sum_{i<j}^{A} v_{i j}$ can be written as $V=$ $\sum_{f=1}^{M_{f}} V_{f}+\sum_{f<g}^{M_{f}} V_{f g}$ where $V_{f}=\sum_{i<j \in f} v_{i j}$ and $V_{f g}=$ $\sum_{i \epsilon f, j \in g, f \neq g} v_{i j}$ are the intrafragment and interfragment components respectively. In the experimental analysis of ref. 1] the only interaction considered among the different fragments is the Coulomb force because of its long range nature. The interfragment nuclear force is thus neglected following the argument that an important nuclear interaction is incompatible with the freeze-out concept. Concerning the evaluation of the intrafragment potential energy $V_{f}=\sum_{i<j \in f} v_{i j}$, this latter is approximated in the experimental analysis [1] by the tabulated ground state nuclear energies. Both these approximations are used in many other thermodynamic studies of multifragmentation [14, 18] and in all macroscopic statistical models 15.

\section{A. Independent Liquid drop approximation}

In order to check the quality of these approximations, we shall use an exact numerical experiment. This restricts studies to classical systems. At variance with nuclear systems which are liquid in their ground states, the ground states of classical models are solid and so present an extra binding. To avoid this difficulty, we may approximate the interaction energy $V$ as a sum of independent liquid drops contributions

$$
Q_{L D}=\sum_{f=1}^{M_{f}} B_{f}=\sum_{f=1}^{M_{f}} a_{V} A_{f}+a_{S} A_{f}^{2 / 3}
$$

where $A_{f}$ is the size of cluster $f$ and $M_{f}$ is the total number of clusters. The parameters $a_{V}, a_{S}$ can then be fitted to reproduce the cluster binding at low but finite temperature to avoid the peculiarities of the ground states of such classical systems.

In this article we present a study based on exact numerical experiments of the 3D Lattice gas model [9]. The ground states of such a model are cubes and, as discussed above, to estimate the internal interaction energy of the liquid fragments avoiding the extra binding of those peculiar cubic configurations we shall use low temperature simulations. Using canonical simulations with temperatures around $1 / 3$ of the critical temperature (or microcanonical ones with energies around $-1.5 \epsilon$ ) leads to $a_{V}=-2.86 \epsilon, a_{S}=2.73 \epsilon$, where $\epsilon$ is the lattice coupling (see discussion of Fig 1).

For comparison the choice $a_{V}=-3 \epsilon, a_{S}=3 \epsilon$ corresponds to large cubic clusters, while $a_{V}=-3.06 \epsilon$, $a_{S}=3.35 \epsilon$ leads to a good description of the zero temperature clusters in the size range $2<A_{f}<60$.

In the following calculations the coefficients $a_{V}=$ $-2.86 \epsilon, a_{S}=2.73 \epsilon$ will be kept constant, and we will come back to the influence of the parameters values in the last section.

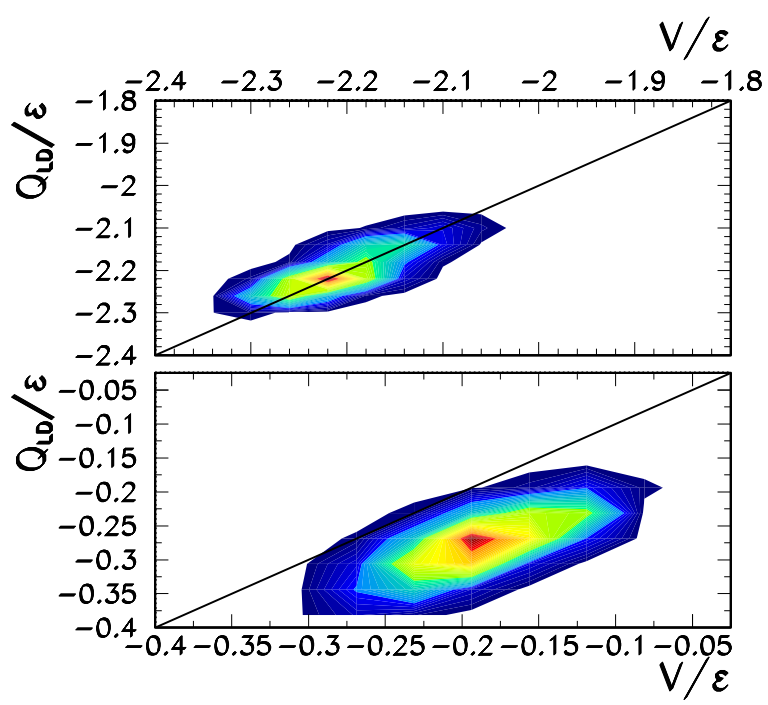

FIG. 1: Correlation between the exact interaction energy $V$ and its approximation from fragment sizes $Q_{L D}$ (see text) at an average density $\rho / \rho_{0}=0.0135$ and a temperature $T / T_{c}=$ 0.29 (upper part), and $T / T_{c}=0.57$ (lower part). The liquid drop parameters for the fragment binding energy are fixed as $a_{V}=-2.86 \epsilon, a_{S}=2.73 \epsilon$. 


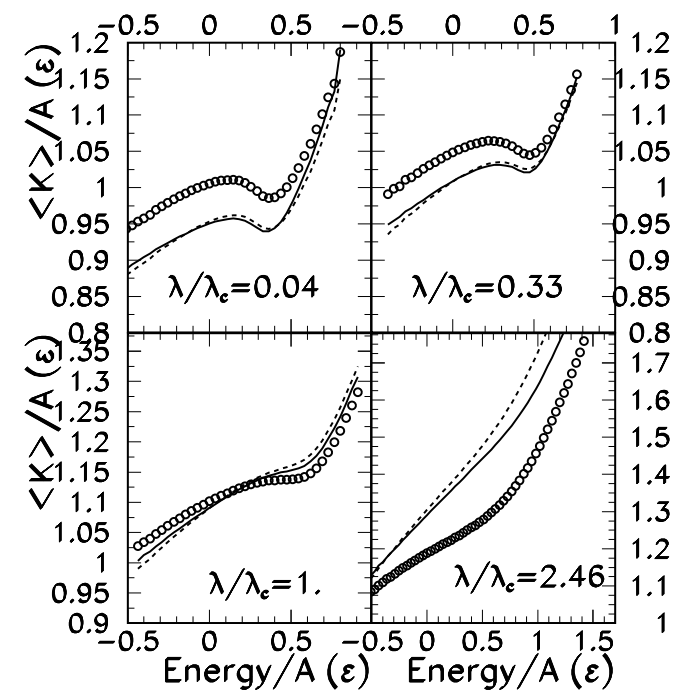

FIG. 2: Reconstruction of the average kinetic energy (solid lines) as a function of total energy from fragment sizes in the Lattice Gas model at different pressures. Dashed lines: liquid drop parameters from table 1. Symbols: liquid drop parameters fixed from the low temperature low density phase

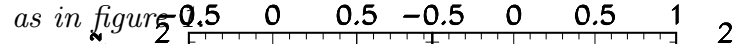

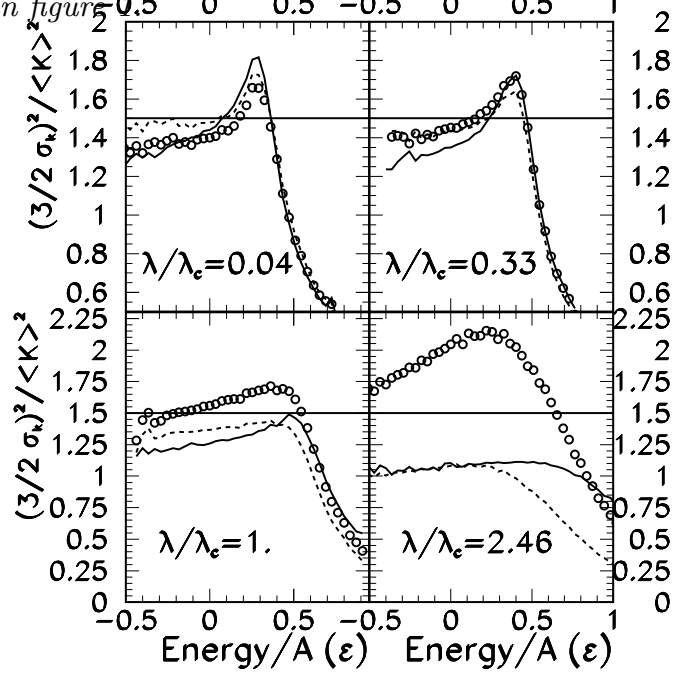

FIG. 3: Reconstruction of the normalized kinetic energy fluctuation (solid lines) as a function of total energy from fragment sizes in the Lattice Gas model at different pressures. Dashed lines: liquid drop parameters from table 1. Symbols: liquid drop parameters fixed from the low temperature low density phase as in figure 1.

We have first tested the accuracy of the approximation (2) for the total interaction energy $V$ in a low density case (108 particles in a cubic box of volume $L^{3}=8000$ ) where the interfragment energies can be safely neglected. The upper part of figure 1 shows the correlation between the exact interaction energy and its independent fragment approximation eq.(2) in a canonical simulation at a temperature $T / T_{c}=0.29$. The corresponding average total energy is $-1.74 \epsilon$, well below the backbending region. The good linearity of the plot (the $Q_{L D} / V$-correlation coefficient is $c=0.77$ ) shows that indeed in such a low density configuration the interaction energy can be calculated within a liquid drop approximation.

Even in the independent fragment picture, the liquid drop coefficients are expected to change with temperature reflecting the internal excitation of the produced clusters. This can be seen in the lower part of figure [1] which shows a calculation for the same very diluted system at a temperature well above the transition temperature. It is clear that the quality of the correlation does not significantly change with the temperature (here the $Q_{L D} / V$-correlation coefficient is $\left.c=0.78\right)$, but the use of the low temperature mass formula leads to a systematic $\approx 20 \%$ overestimation of the fragment binding that can affect the thermodynamic analyses.

\section{B. The choice of the statistical ensemble}

This point should be further explored looking at direct effects of the considered approximation on thermodynamical quantities, i.e. on ensemble averaged observables such as averages and variances. Since the different statistical ensembles are not equivalent in finite systems, the statistical ensemble has to be specified. In the following we have chosen to perform calculations in the microcanonical "isobar" ensemble characterized by the two state variables $(E, \lambda)$, the total energy and the Lagrange multiplier imposing the average volume $V$, respectively [9]. Statistical averages are calculated as

$$
\langle A\rangle_{E, \lambda}=\frac{\sum_{(n)} A^{(n)} \exp \left(-\beta E^{(n)}-\lambda V^{(n)}\right) \delta\left(E^{(n)}-E\right)}{\sum_{(n)} \exp \left(-\beta E^{(n)}-\lambda V^{(n)}\right) \delta\left(E^{(n)}-E\right)}
$$

where $A$ is a generic observable ( $A=K$ allows to compute the microcanonical temperature, $A=K^{2}$ provides the heat capacity), the sum runs over the system microstates, and the average volume is defined through the one body observable

$$
V=\frac{4 \pi}{3 L^{3}} \sum_{i=1}^{L^{3}} r_{i}^{3} n_{i}
$$
site.

where $n_{i}=0,1$ is the occupation of the $i-t h$ lattice

Different reasons motivate the choice of this ensemble. First, this is the ensemble in which the liquid gas phase transition is associated to a negative heat capacity up to the critical point 19. Moreover in the actual analysis of heavy ion experiments data are sorted in excitation energy bins, i.e. approximate realizations of microcanonical ensembles. On the other side the system extension is only imposed by the freeze out requirement without any boundary condition. We have recently shown that such a physical situation is accounted in a thermostatistical coherent way only if the system size is imposed through 
a lagrange parameter $\lambda$, with $P=\lambda / \beta$ having the dimension (and the physical role) of a constraining pressure 20$]$. Finally it is interesting to notice that if $\lambda=0$ this "isobar" ensemble is equivalent to the isochore ensemble used in section $\amalg$ if the volume is large enough such that the boundary conditions become irrelevant.

\section{Averages and fluctuations}

Figure 2 shows the estimated average kinetic energy $E-\sum_{f} B_{f}$ as a function of the total energy with different constraints on the system volume [9], corresponding to different pressures. The shape of the exact caloric curve (solid line) is nicely reproduced by the independent "cold" liquid drop approximation (open symbols) for all volume constraints. Using the temperature backbending to define the phase transition, we see that the coexistence zone as well as the critical point can be well estimated from the unique knowledge of the fragment partitions. However, the actual value of the temperature shows a systematic shift. The fact that this shift is also present in the pure high temperature low density gas phase (figure 1) suggests that it may be due to a temperature dependence of the fragment internal energy which is notaccounted fon

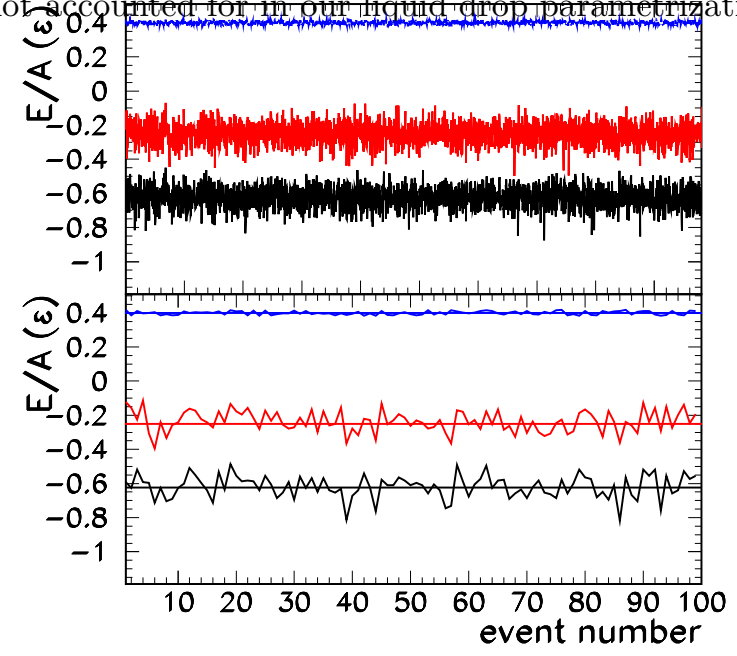

FIG. 4: Upper panel: partial energies of an ensemble of events at a pressure $\lambda / \lambda_{c}=.33$ and energy $.38 \epsilon<E_{\text {tot }} / N<.42 \epsilon$ in the middle of the coexistence region. From bottom to top: -interaction energy per particle; -its independent fragment approximation (LD parameters as in figure 1) (shifted by 0.4); -total energy per particle. The horizontal lines give the average values of the corresponding energies. Lower panel: same as the upper panel on an extended scale.

The effect on partial energy fluctuations is shown in figure 3 for the same thermodynamic conditions as in figure 2] We can see that the fluctuations tend to be overestimated, but since the bias on the average value goes in the same direction, the normalized fluctuations are still reasonably reproduced almost up to the critical point.

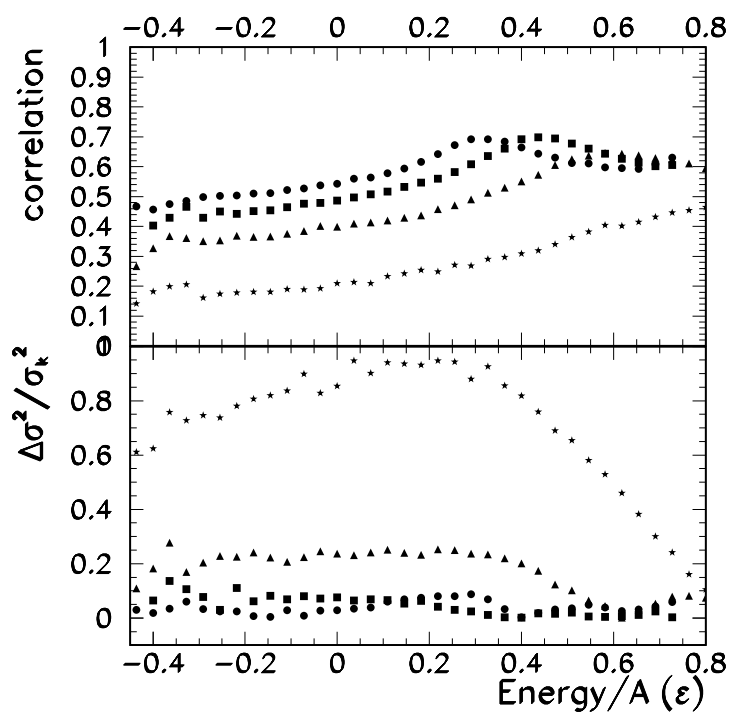

FIG. 5: $Q_{L D} / V$-correlation coefficient (upper part) and percentage error $\left|\sigma_{Q}^{2}-\sigma_{K}^{2}\right| / \sigma_{K}^{2}$ on the partial energy fluctuation from figure 3 (lower part). The liquid drop parameters are fixed as $a_{V}=-2.86 \epsilon, a_{S}=2.73 \epsilon$. Black points: $\lambda / \lambda_{c}=.04$, squares: $\lambda / \lambda_{c}=.33$, triangles: $\lambda / \lambda_{c}=1$, stars: $\lambda / \lambda_{c}=2.46$.

Even if the quantitative study of a statistical ensemble should be based on ensemble averages such as the one presented in the previous figures, it is instructive to look at a set of events corresponding to one of the above cases as shown in Fig. 4 This figure illustrates the fact that the actual interaction energy and its independent liquiddrop approximation have similar properties, averages and fluctuations. Moreover, the extended scale part shows that the two quantities are correlated.

\section{Errors and correlations}

In order to quantify the quality of the liquid drop approximation let us look at ensemble averaged quantities which are indeed the information used to infer thermodynamical properties. One of the most important observables is the kinetic energy fluctuation. The corresponding percentage error $\left|\sigma_{Q}^{2}-\sigma_{K}^{2}\right| / \sigma_{K}^{2}$ is shown in the lower part of figure [5. One observes that the error increases with the pressure but remains low for all energies up to the critical pressure. This error is below $10 \%$ up to $1 / 3$ of the critical pressure and below $20 \%$ at the critical pressure. Moreover, when used to compute the heat capacity this error is partly compensated by the similar error on the kinetic energy used to deduce the temperature. This explains the good accuracy observed for the liquid drop approximation up to rather high temperatures and pressures.

This good accuracy of the ensemble averaged quantities does not imply nor require a similar accuracy on an 
event by event basis. In fact one expects stronger deviations when looking at a single event. One way to study this event by event accuracy of the liquid drop approximation is to study the correlation coefficient $c$. However, this is only a part of the discussion since the correlation coefficient is not sensitive to the magnitude of the fluctuation and only characterizes the link between the independent liquid drop approximation and the actual internal energy. In the considered case, this implies that by construction the correlation coefficient $c$ is independent of the value of the liquid drop parameters. The global trend of the correlation coefficient with pressure and energy is presented in the upper part of figure 5. We can see that the best correlation is systematically observed around the fluctuation peak, where the size distribution is the broadest. At subcritical pressures, $c$ always exceeds 0.4 , while the correlation decreases to around 0.2 in the supercritical regime.

It should be stressed that the idea to use, in equation (1), the first moments (averages and fluctuations) of the event distribution was indeed to overcome the difficulty to get accurate information on an event by event basis. Though the best correlation obviously leads to the smallest error, we can see that the relation between $c$ and $\Delta \sigma$ is not trivial. In particular a value of $c$ exceeding 0.4 comes out to be enough to keep the error below the $15 \%$ level, independent of the energy.

It is also interesting to remark that the correlation coefficients shown in figure 5 are systematically lower than the ones shown in the calculations of figure 11 This is another illustration of the non equivalence of statistical ensembles: in the canonical case the distributions are wider than in the microcanonical one allowing a better correlation.

\section{E. Beyond the fixed cluster energy approximation}

The failure at increasing pressure shown by fig 3 is interesting. One may wonder whether the lack of reproduction is due to the breaking down of the independent fragment approximation [5] in dense media, or whether the configurational contribution to the internal fragment excitation energy has to be taken into account by a proper redefinition of the mass formula. To answer to this question we have allowed a free variation of the liquid drop parameters according to table 1 . The result, shown by the dashed lines in figs 23, is that both the caloric curve and the fluctuations can be very precisely reproduced by the independent fragment approximation in a wide range of temperatures and pressures if the volume and surface coefficients are allowed to decrease with increasing excitation. Only at very high pressures and temperature, above the critical point, the approximation appears to clearly break down: in this dense configuration the objects identified as "fragments" by the cluster recognition algorithm have certainly little to share with physical isolated liquid drops. Indeed in this density regime the $\mathrm{Q}$ value eq.(2) is very poorly correlated with the interaction energy (see figure 5).

\begin{tabular}{|c|c|c|c|}
\hline$\frac{\lambda}{\lambda_{c}}$ & $\frac{\langle V\rangle_{c}}{\left\langle V>_{c}\right.}$ & $\frac{a_{v}}{\epsilon}$ & $\frac{a_{s}}{\epsilon}$ \\
\hline 0.04 & 2.00 & -2.76 & 2.66 \\
\hline 0.33 & 1.69 & -2.74 & 2.61 \\
\hline 1.00 & 1.00 & -2.72 & 2.53 \\
\hline 2.46 & 0.53 & -2.64 & 2.18 \\
\hline
\end{tabular}

TABLE I: Volume $a_{V}$ and surface $a_{S}$ effective liquid drop parameters (see text) allowing to reproduce the average canonical configurational energy of 216 Lattice Gas particles at the temperature corresponding to the maximal energy fluctuations, for different pressures normalized to the critical pressure. The average volume occupied by the system divided by the critical volume is also given.

\section{DISCUSSION AND POSSIBLE EXPERIMENTAL IMPLICATIONS}

Let us now turn to the possible implications of these findings to heavy ion collisions experiments, and particularly to the determination of the heat capacity from the fluctuations of asymptotic detected partitions.

In the application of eq11 to the analysis of multifragmentation data several difficulties arise that have to be considered.

First of all in the experimental case the only information available on the system is given by its cluster properties (sizes and kinetic energies). This means that the total energy of the system is not evaluated from its microscopic constituents, but is also estimated from the measured kinetic energies and fragment sizes as $E_{\text {tot }}=Q_{L D}+K$.

Moreover both $Q_{L D}$ and $K$ are time dependent variables, and in the data analysis the quantities at freeze out are extrapolated from the asymptotic ones solely correcting for secondary evaporation and Coulomb repulsion. In particular the configurational energy functional associated to each fragment at freeze out is assumed to be the one associated to $T=0$ and is not pressure dependent. As we have seen in the previous section, this assumption is not valid for the Lattice Gas model: the presence of a cubic ground state in this model leads to a mass formula that is not adequate to reproduce the average cluster energy in the liquid phase. The difference in energy is small compared to the liquid-vapour latent heat, however we have seen that it can have important effects in the calculation of the observables. This difficulty in principle should not arise in nuclear physics where clusters are already liquid at $\mathrm{T}=0$, however one may ask how much a possible modification with temperature and pressure of the fragments energetics would influence the experimental analysis.

Finally the assumption is explicitly made that at the 
freeze out time fragment partitions essentially reflect thermal equilibrium.

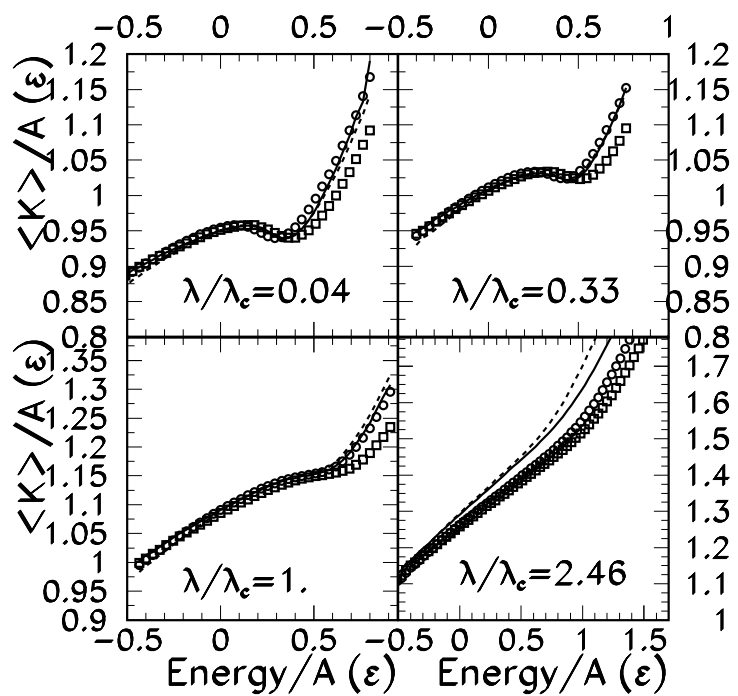

FIG. 6: Same as figure above, but including the effect of the calorimetric estimation of total energy (see text). Squares: zero temperature liquid drop parameters $a_{v}=-3 \epsilon, a_{s}=3 \epsilon$.

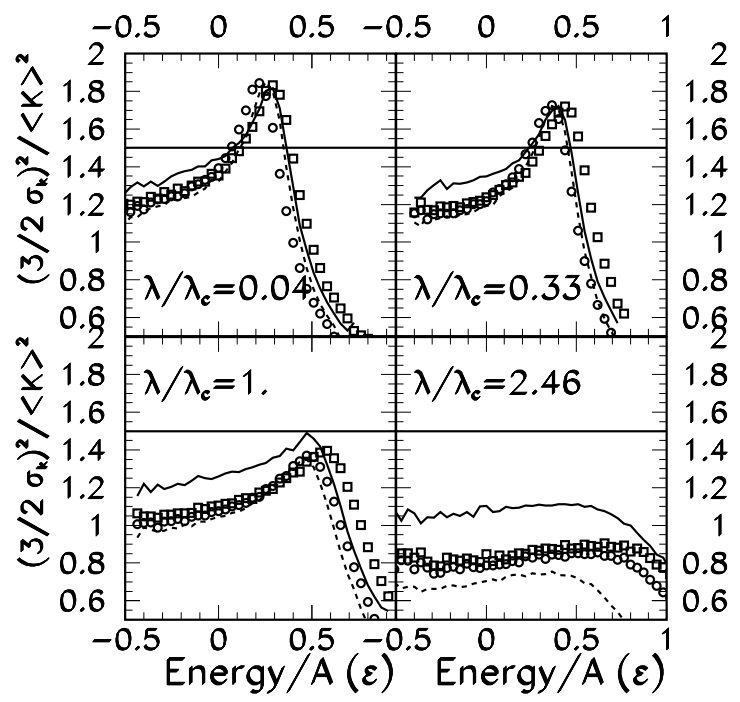

FIG. 7: Same as figure 3 above, but including the effect of the calorimetric estimation of total energy (see text). Squares: zero temperature liquid drop parameters $a_{v}=-3 \epsilon, a_{s}=3 \epsilon$.

\section{A. The thermodynamics of a gas of clusters}

Let us leave the difficult problem of equilibrium and time dependence to the next subsection and start addressing the question of the quality of reconstruction of the thermodynamics of freeze-out, using accessible information (fragment sizes and total kinetic energies). In this matter, equilibrium studies provide a valuable testing ground, if the studied thermodynamic conditions correspond to the freeze-out configurations of the model.

To quantify the uncertainty of the heat capacity reconstruction from the fragment information only, we have introduced reconstructed microcanonical statistical ensembles defined by the total energy constraint $E_{c a l}=$ $K+Q_{L D}$ at different pressures and with different prescriptions for the liquid drop parameters. Figures 6 and 7 show the resulting first and second moment of the $K$ distribution in bins of $E_{c a l}$. We can see that the systematic bias observed in fig 2 disappears if the same prescription for the interaction energy is used both for the fluctuation and for the total energy of the system. Up to the critical point, the precise parametrization of the liquid drop energy does not change the results dramatically. In particular the zero temperature cubic solid cluster prescription $a_{v}=-3 \epsilon, a_{s}=3 \epsilon$ induces a spurious shift towards higher energies, but does not induce extra fluctuations.

These results may be qualitatively understood as follows. The error in the estimation of $V$ through $Q_{L D}$ in each event of a set at a given total physical energy induces an overall bias in the evaluation of $\left\langle E_{t o t}\right\rangle$, but also and more important, a spread in the total energy estimation. Both these effects are especially important at high pressures, where the correlation coefficient between $Q_{L D}$ and $V$ is low (see fig 5 ). The systematic bias in the estimation of the average total energy depends directly on the value chosen for the liquid drop parameters, and leads to the shift towards higher energy observed in figs 6 6] The width of the $E_{c a l}$ distribution for each value of $E_{t o t}$ is at the origin of the overestimation of the partial energy fluctuations observed in fig 3 When events are analyzed in bins of total estimated energy, these spurious energy fluctuations do not contribute any more to the width of the $K$ distribution. The remaining effects are due to the mixing in a given $E_{c a l}$ bin of events coming from different physical $E_{t o t}$ values. Since this mixing is approximately symmetric in energy, the average $\left\langle E_{k}\right\rangle$ appears to be not much affected and the main effect of mixing is to flatten out the fluctuation curve. This is very similar to the results already reported for the analysis of events produced in macroscopic statistical models (SMM[11] and SIMON[21]).

From figs 6 and 7 we can also see that, when calorimetry is taken into account, it is not possible any more to tune the liquid drop parameters at high pressure such as to reproduce at the same time the correct average energy and fluctuation. This confirms again that the independent fragment hypothesis breaks down in the supercritical regime.

The error on the fluctuation estimation is reported in fig 8 for all the considered pressures and energies, using the fixed low temperature liquid drop parameters $a_{V}=$ $-2.86 \epsilon, a_{S}=2.73 \epsilon$. The fact of taking into account the calorimetric shift does not increase this systematic error 


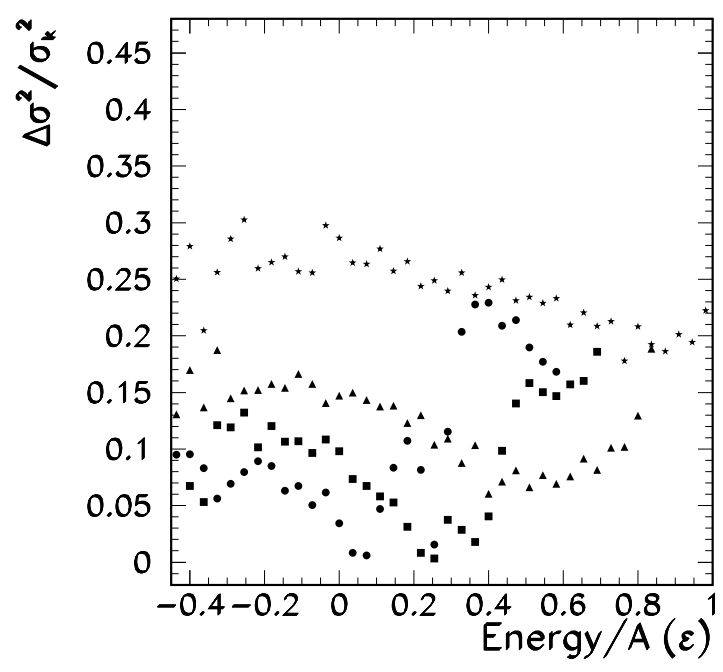

FIG. 8: Percentage error $\left|\sigma_{Q}^{2}-\sigma_{K}^{2}\right| / \sigma_{K}^{2}$ on the partial energy fluctuation from figure 7 The liquid drop parameters are fixed as $a_{V}=-2.86 \epsilon, a_{S}=2.73 \epsilon$. Black points: $\lambda / \lambda_{c}=.04$, squares: $\lambda / \lambda_{c}=.33$, triangles: $\lambda / \lambda_{c}=1$, stars: $\lambda / \lambda_{c}=2.46$.

above the $20 \%$ level at subcritical pressure.

The results of figs 6.7 suggest that if the freeze-out density is low enough, then the thermodynamic properties of the system can be deduced from the fragment partitions using the independent fragment model with a fragment energy approximated by its low temperature binding. In the Lattice-gas model the validity condition of this simple approximation happens to be not so restrictive since it corresponds to an average volume $V \gtrsim 1.5 V_{c}$. If the freeze out density is higher, in the lattice-gas model the independent fragment approximation tends to break down, and both the temperature and the fluctuation are systematically underestimated.

\section{B. The influence of dynamical and quantum effects}

When dealing with reactions and trying to extract thermodynamic information, the first question is the relevance of the freeze-out and equilibrium concepts at specific stages of the collision. From the theoretical point of view, this complex question, which is strongly debated since more then a decade, can only be addressed by complete simulations of the reaction and critical analysis of the resulting time dependence. Such studies require to go beyond the equilibrium models used in the present article as well as in ref. [5]. In a recent dynamical calculation with the Lennard-Jones hamiltonian, A.Chernomoretz et al. [10] have addressed this question by looking at the time evolution of different observables for a system initially thermalized in a confined box and subsequently freely evolving in the vacuum. Almost independent of the initial energy and density, the average configurational en- ergy and the associated fluctuations turn out to freeze when the density of the system is of the order of $\rho \approx 0.03$ in Lennard-Jones units, a region of the phase diagram well below the critical point 2]. This study, thus, implies that only such diluted stages of the reaction can be reconstructed from the asymptotic ("experimental") information. However, it is important to stress that the density value at freeze out is a model dependent quantity and in particular it is correlated with the range of the force. In the study of ref. [10], the configurations at freeze-out are also characterized by an interfragment energy sufficiently small $\sum_{f<g}^{M_{f}} V_{f g}(t) \approx 0$ for fragment partitions to be essentially frozen. Conversely to the absolute value of the density, this condition might be a more robust definition of freeze out. It is also a first argument in favor of an independent fragment approximation.

Another consequence of the time dependence of a collisional process is that the freeze out configuration may not be sufficiently close to a thermodynamic equilibrium to be described with statistical tools 2, 22]. The statistical nature of freeze-out is indeed the key point in order to interpret the reconstructed fluctuation in terms of heat capacity. To estimate the distortions due to the out of equilibrium component one needs to know how much the averages and fluctuations deviate from the equilibrium values. Different verifications have been performed to estabilish this point [11] in the experimental analyses of collisional data. In particular, the observation of the same behavior with different entrance channels is an interesting argument in favor of being close to an equilibrium. From a theoretical point of view, this fundamental open question can only be addressed through dynamical approaches and cannot be answered from statistical calculations as the one presented here.

Finally it is important to stress that the results of classical models like the Lattice Gas (or the Lennard Jones analyzed in refs. [5], 10]) cannot be quantitatively applied to nuclear data because of the complete lack of quantum effects. The ground state properties are not the only point where quantum effects are expected to play an important role. The actual value of the density at freeze out of a nuclear system can be very different from the one estimated from classical calculations [10], and the same is true for the limiting density that can be described through the independent fragment approximation derived in this work. The consistent inclusion of quantum effects in the statistical analyses of nuclear data is an ambitious program that is only in its infancy 19. and constitutes one of the greatest theoretical challenges of heavy ion collisions in the next decades.

\section{CONCLUSION}

To summarize, in this paper we have reconstructed configurational energy fluctuations from the fragment partitions of a Lattice Gas model. As far as the first and 
second moments of collective observables are concerned, dilute systems in thermodynamic equilibrium can be accurately approximated by an ensemble of independent fragments. A unique liquid drop parametrization for the fragment binding energies is able to describe the thermodynamics of the system independent of the deposited energy or temperature. A modification of the order of $10 \%$ of the liquid drop parameters does not modify the temperature and fluctuation in a sizeable way, if the same parametrization is consistently employed for the determination both of the fluctuation and of the total energy. For pressures of the order or above the critical point, the independent fragment approximation tends to break down leading to a systematic underestimation of temperatures and fluctuations at the $30 \%$ level.

\section{Acknowledgments}

Discussions with F.Cannata are gratefully acknowledged.
[1] M.D'Agostino et al., Phys.Lett. B473,219(2000).

[2] P.Balenzuela, A.Chernomoretz and C.O.Dorso, Phys.Rev. C66,24613(2002).

[3] L. G. Moretto, J. B. Elliott, L. Phair, and G. J. Wozniak Phys.Rev. C66,041601(2002); F. Gulminelli and Ph. Chomaz, Phys.Rev. C69,069801(2004); L. G. Moretto, J. B. Elliott, L. Phair, and G. J. Wozniak Phys.Rev. C69,069802(2004).

[4] W.Thirring, H.Narnhofer and H.A.Posch, Phys.Rev.Lett. 91,130601(2003)

[5] X.Campi, H.Krivine, E.Plagnol and N.Sator, arXiv:nucl-th/0406056 2 .

[6] F. Gobet, B. Farizon, M. Farizon, M. J. Gaillard, J. P. Buchet, M. Carr, P. Scheier, and T. D. Mrk Phys.Rev.Lett. 89,183403(2002).

[7] Ph. Chomaz, F. Gulminelli, Physica A330,451(2003); D. H. E. Gross, "Microcanonical thermodynamics: phase transitions in finite systems", Lecture notes in Physics vol. 66, World Scientific (2001).

[8] Ph. Chomaz and F. Gulminelli, Nucl.Phys. A647,153 (1999).

[9] F. Gulminelli, Ph. Chomaz, V.Duflot, Phys.Rev.Lett. $85,3587(2000)$.

[10] A. Chernomoretz, F. Gulminelli, M. J. Ison, and C. O. Dorso Phys.Rev. C69,034610(2004).
[11] M. D'Agostino et al., Nucl.Phys. A699,795(2002); Nucl.Phys.A734,512(2004).

[12] T.L. Hill, J.Chem.Phys. 23,617(1955).

[13] Ph.Chomaz, F.Gulminelli and M.D'Agostino, arXiv:nucl-th/0407047

[14] J.B.Elliott et al., Phys.Rev.Lett. 88,042701(2002); Phys.Rev. C67,024609(2003).

[15] J. P. Bondorf et al., Phys. Rep. 257,133(1995); D.H.E.Gross, Phys.Rep. 279,119(1997).

[16] J.L.Lebowitz, J.K.Percus and L.Verlet, Phys.Rev. $153,250(1967)$.

[17] It is worthwhile noticing that, if eq. (1) has been derived in ref. [16] only for the isochore ensemble, the more general derivation of ref. 8] does not suffer of this limitation.

[18] R.P.Scharenberg et al., Phys.Rev.C64 054602, (2001).

[19] Ph.Chomaz and F. Gulminelli, "Phase transitions in finite systems", in 'Dynamics and Thermodynamics of Systems with Long Range Interactions', Lecture Notes in Physics vol.602, Springer (2002).

[20] Ph.Chomaz, F.Gulminelli and O.Juillet, cond-mat/0412475 and Annals of Physics, in press.

[21] E.Vient et al.,Nucl.Phys.A700 (2002)555.

[22] J.Colin et al., Phys.Rev.C67 (2003)064603; B.Borderie et al., Phys.Rev.Lett.86 (2001) 3252. 